Technical Progress on

\title{
INNOVATIVE ELECTROMAGNETIC SENSORS FOR PIPELINE CRAWLERS
}

\author{
Type of Report: Technical Progress Report \\ Reporting Period Start Date: October 1, 2004 \\ Reporting Period End Date: March 31, 2005 \\ by \\ J. Bruce Nestleroth and Richard J. Davis
}

Submitted

May 23, 2005

NETL Award No. DE-FC26-03NT41881

PRCI Contract No. PR-003-03155

Battelle

505 King Avenue

Columbus, Ohio 43201 


\section{Notice}

This report was prepared as an account of work sponsored by an agency of the United States Government. Neither the United States Government nor any agency thereof, nor any of their employees, makes any warranty, express or implied, or assumes any legal liability or responsibility for the accuracy, completeness, or usefulness of any information, apparatus, product, or process disclosed, or represents that its use would not infringe privately owned rights. Reference herein to any specific commercial product, process, or service by trade name, trademark, manufacturer, or otherwise does not necessarily constitute or imply its endorsement, recommendation, or favoring by the United States Government or any agency thereof. The views and opinions of authors expressed herein do not necessarily state or reflect those of the United States Government or any agency thereof.

Neither Battelle, nor any person acting on their behalf:

(1) Makes any warranty or representation, expressed or implied, with respect to the accuracy, completeness, or usefulness of any information contained in this report or that the use of any information, apparatus, method, or process disclosed in this report may not infringe privately owned rights.

(2) Assumes any liabilities with the respect to the use of, or for damages resulting from the use of any information, apparatus, method or process disclosed in this report. 


\begin{abstract}
Internal inspection of pipelines is an important tool for ensuring safe and reliable delivery of fossil energy products. Current inspection systems that are propelled through the pipeline by the product flow cannot be used to inspect all pipelines because of the various physical barriers they encounter. Recent development efforts include a new generation of powered inspection platforms that crawl slowly inside a pipeline and are able to maneuver past the physical barriers that can limit inspection. At Battelle, innovative electromagnetic sensors are being designed and tested for these new pipeline crawlers. The various sensor types can be used to assess a wide range of pipeline anomalies including corrosion, mechanical damage, and cracks.

The Applied Energy Systems Group at Battelle is in the second year of work on a projected three-year development effort. In the first year, two innovative electromagnetic inspection technologies were designed and tested. Both were based on moving high-strength permanent magnets to generate inspection energy. One system involved translating permanent magnets towards the pipe. A pulse of electric current would be induced in the pipe to oppose the magnetization according to Lenz's Law. The decay of this pulse would indicate the presence of defects in the pipe wall. This inspection method is similar to pulsed eddy current inspection methods, with the fundamental difference being the manner in which the current is generated. Details of this development effort were reported in the first semiannual report on this project. The second inspection methodology is based on rotating permanent magnets. The rotating exciter unit produces strong eddy currents in the pipe wall. At distances of a pipe diameter or more from the rotating exciter, the currents flow circumferentially. These circumferential currents are deflected by pipeline defects such as corrosion and axially aligned cracks. Simple sensors are used to detect the change in current densities in the pipe wall. The second semiannual report on this project reported on experimental and modeling results. The results showed that the rotating system was more adaptable to pipeline inspection and therefore only this system will be carried into the second year of the sensor development.

In this third reporting period, the rotating system inspection was further developed. Since this is a new inspection modality without published fundamentals to build upon, basic analytical and experimental investigations were performed. A closed form equation for designing rotating exciters and positioning sensors was derived from fundamental principles. Also signal processing methods were investigated for detection and assessment of pipeline anomalies. A lock in amplifier approach was chosen as the method for detecting the signals. Finally, mechanical implementations for passing tight restrictions such as plug valves were investigated. This inspection concept is new and unique; a United States patent application has been submitted.
\end{abstract}


This page intentionally blank. 


\section{Table of Contents}

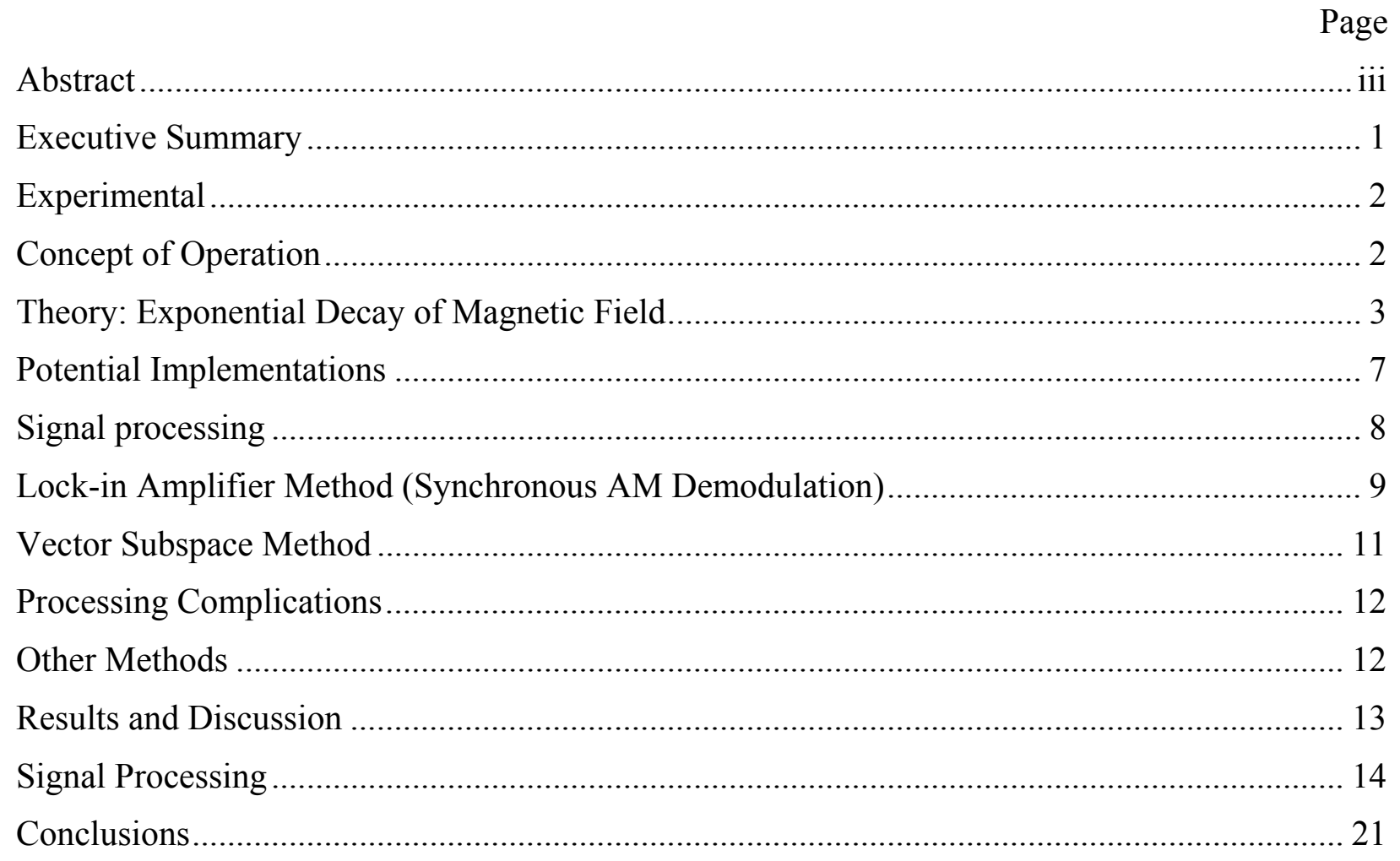

\section{List of Figures}

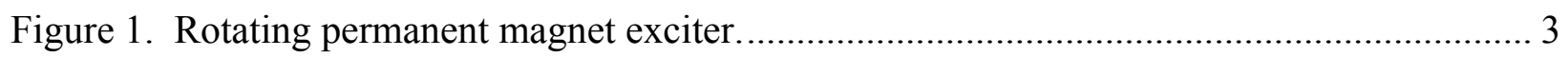

Figure 2. A comparison of the first-order estimate, modeling and experimental results............... 7

Figure 3. A telescopic magnet bar that enables the magnets to retract as they pass over

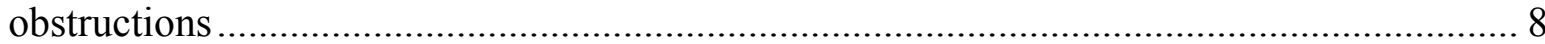

Figure 4. A hinged structure that enables the magnets to retract as they pass over obstructions. . 8

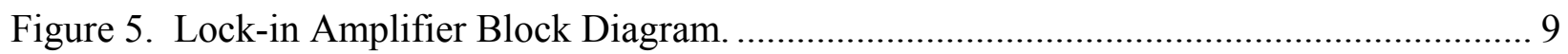

Figure 6. Experimental results showing the decay rate is related to both pipe diameter and

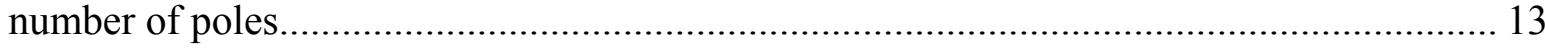

Figure 7. Simulation 1 Results............................................................................................ 15

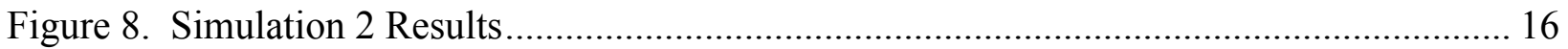

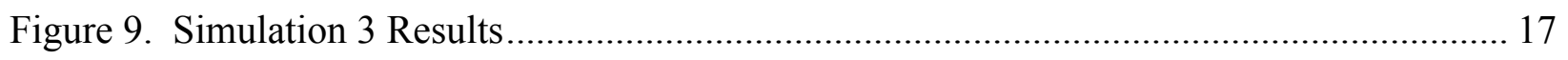

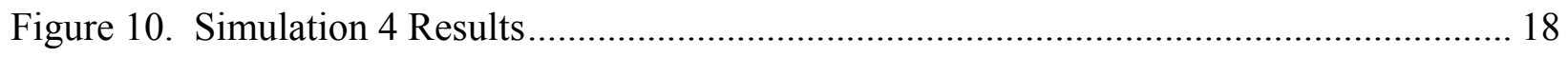

Figure 11. Simulation 5 Results......................................................................................... 19

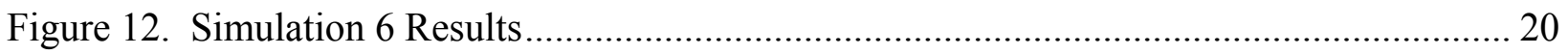




\section{Table of Contents (continued) List of Tables}

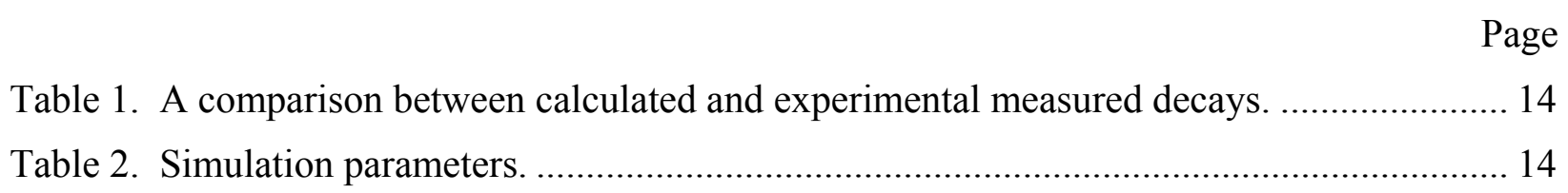




\section{Executive Summary}

The basic requirements for sensor systems designed for installation on pipeline crawlers include small physical size and weight as well as low electrical power consumption. Magnetic flux leakage (MFL), the most common technology used to inspect pipelines, is difficult to implement on autonomous crawler systems because the sensors are large and heavy. In addition, although MFL sensors are slow moving, they require significant power to measure the flux leaking from defects and are not able to detect all defect types. Among alternative approaches, small and light sensor technologies have shown promise but implementation attempts have been thwarted by high speed and long distance requirements, factors that are not as restrictive for crawler-based inspection systems.

The objective of this project is to develop electromagnetic sensors for mounting on a crawling inspection platform that moves slowly through the pipeline interior. These sensors will be used to assess a wide range of pipeline conditions including corrosion (pitting, patches, and general), mechanical damage, cracking, and seam weld defects. The sensors must be light weight and low drag to minimize propulsion requirements of the crawler platform. In addition, the sensors will require minimal power for excitation of interrogating energy and sensor current for anomaly detection.

The focus of work in the first year of this project has been on prototype development. The first semiannual report covered the development of a translating permanent magnet induced pulsed eddy current system. The second semiannual report covered the development of the rotating permanent magnet exciter to induce eddy currents for the inspection of pipelines. Since the results showed that the rotating system was more adaptable to pipeline inspection, only this system will be carried into the second year of the sensor development.

In this third reporting period, the rotating system inspection was further developed. Since this is a new inspection modality without published fundamentals to build upon, basic analytical and experimental investigations were performed. A closed form equation for designing rotating exciters and positioning sensors was derived from fundamental principles. Also signal processing methods were investigated for detection and assessment of pipeline anomalies. A lock in amplifier approach was chosen as the method for detecting the signals. Finally, mechanical implementations for passing tight restrictions such as plug valves were investigated. This inspection concept is new and unique; a United States patent application has been submitted. 


\section{Experimental}

A typical approach to nondestructive inspection of materials involves injecting uniform energy into an object. An anomaly or defect disrupts this uniformity. Sensors are used to detect a change in the uniformity and thus the anomaly or defect. Radiography and magnetic flux leakage inspection are common examples of this approach. In radiography, anomalies are detected when more of the incident X-ray energy passes thru the material. A film or charge coupled device placed on the opposite side of the material as the source can be used to detect this change in absorption. In magnetic flux leakage, anomalies are detected when the uniform magnetic field established in the material is disturbed. Magnetic field sensors such as Hall Effect sensors or moving coils detect the local change in magnetic fields due to anomalies. This third semiannual report covers the basic analytical and experimental investigations of the rotating permanent magnet exciter to induce uniform eddy currents for the inspection of pipelines.

\section{Concept of Operation}

A new electromagnetic approach for pipes and tubes has been developed. The method uses rotating permanent magnets to produce an alternating electrical current flowing in the circumferential direction. Figure 1 is a cutaway drawing showing the in-pipe positioning of a rotating permanent magnet exciter, illustrating a concept that has the potential to induce strong eddy currents in the pipe wall. This approach uses alternating $\mathrm{N}$ and $\mathrm{S}$ poles rotating around a shaft, in contrast to traditional eddy current systems, which use a coaxial coil in the pipe that is driven by a sinusoidal current. The power needed by an exciting coil to produce a detectable signal can be significant. This may limit implementation to tethered systems, short-run crawlers, and systems with onboard power generation. 


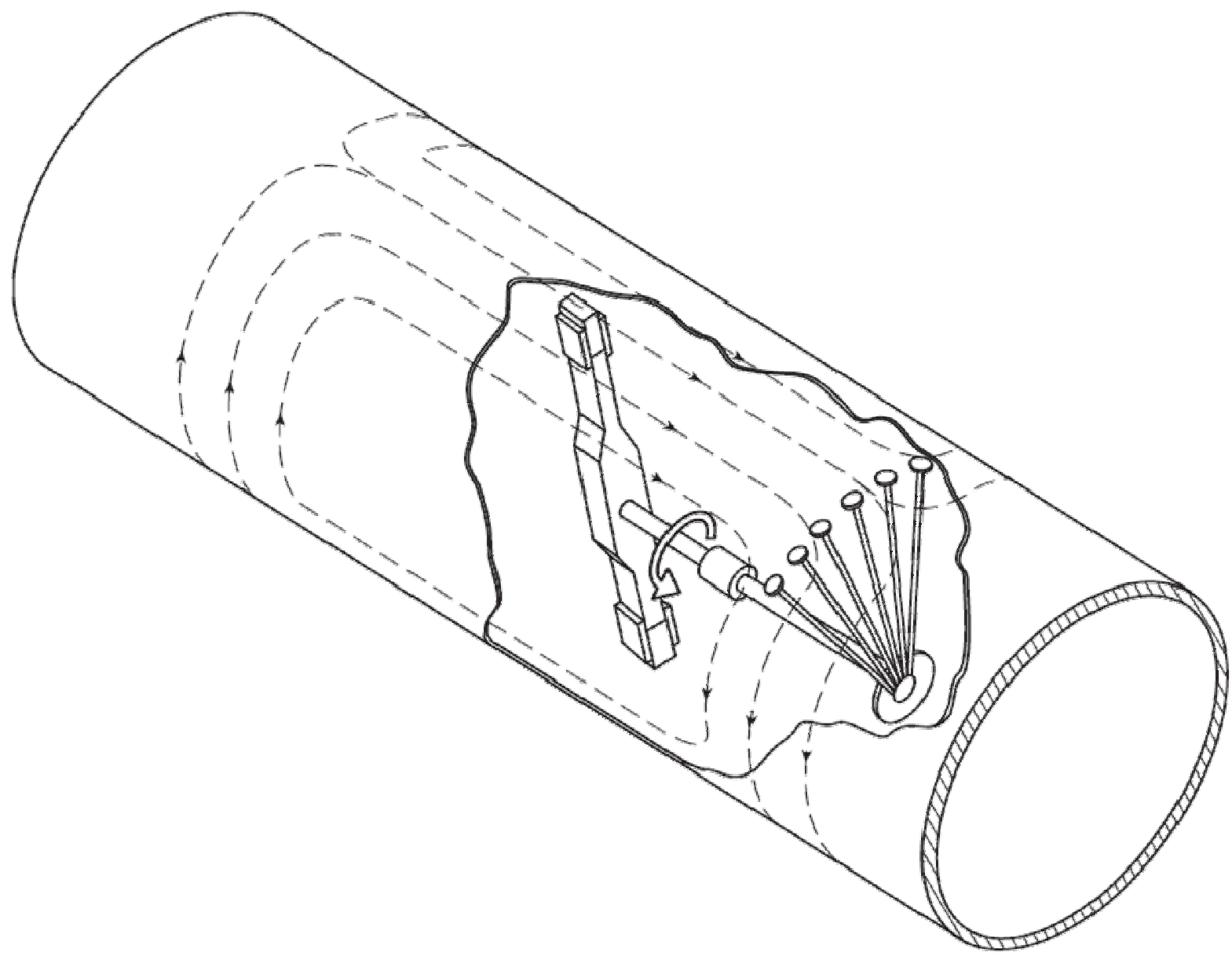

Figure 1. Rotating permanent magnet exciter.

\section{Theory: Exponential Decay of Magnetic Field}

A first order approximation of the field behavior in the rotating magnet system can be obtained through Ampere's Law and the Law of Charge Conservation. Ampere's Law can be written as:

$$
\nabla \times \vec{B}=4 \pi \mu \vec{J}
$$

where $\mathrm{B}$ is the magnetic field, $\mu$ is the magnetic permeability, and $\mathrm{J}$ is the induced current density. This equation relates the behavior of the magnetic field and the induced current density. The Law of Charge Conservation can be written as

$$
\nabla \bullet \vec{J}=0
$$

since there is no build-up of charge here. This equation states that current flows in loops, not segments.

The induced current density can be written as follows:

$$
\vec{J}=\sigma \vec{E}=\sigma \vec{v} \times \vec{B}
$$


where the first part is Ohm's Law and the second is the Lorentz Force with $v$ the velocity of the pipe with respect to the rotating magnetizer and $\sigma$ the conductivity of the pipe. This equation describes the velocity induced eddy currents. Note that this equation suggests that the induced current density is always perpendicular to the velocity. However, because the current density has no divergence (Equation 2), this will not always be the case. For the rotating magnetizer system, the velocity of the pipe wall with respect to the magnetizer is

$$
\vec{v}=-\vec{\omega} \times \vec{r}
$$

where $\omega$ is the rotational speed and $r$ is the radius of the pipe wall. Near the pole piece of the magnetizer (the source), the majority of the field is radial and the velocity is circumferential, so the eddy currents would be axial

$$
J_{Z}=\sigma v B_{R}
$$

Equation 2 can be expanded to first order if we approximate the induced current density as a two-dimensional planar flow independent of pipe wall thickness. In this approximation, Equation 2 can be rewritten in cylindrical coordinates as

$$
\frac{\partial J_{Z}}{\partial z}=-\frac{\partial J_{\phi}}{r \partial \phi}
$$

where the subscripts $z$ and $\varphi$ refer to the axial and circumferential coordinates, respectively. To solve this problem for the behavior of the magnetic fields, a relationship between $J_{Z}$ and $J_{\varphi}$ must be known.

Because of symmetry, the problem can be circumferentially sectioned into $n$ parts, where $n$ is the number of pole pieces in the magnetizer (of course, $n=2,4,6 \ldots$ ). Since at each boundary's interface, the symmetry is mirrored, only one symmetry section needs to be solved. The others are mirrored images of this result. A symmetry section is centered at a pole piece and ranges circumferentially between the angles of $\pm \pi / \mathrm{n}$. For a two pole magnetizer, the symmetry section is $\pm 90^{\circ}$.

To obtain the relationship between the two eddy current components, their circumferential distribution within a symmetry section must be estimated. Since Equation 2 forces the current to flow in loops the following can be predicted. Based on the physics of the problem and assuming a two-dimensional planar flow, the value of $\mathrm{J}_{\varphi}$ at the ends $\left(\varphi= \pm \Phi_{\mathrm{E}}\right)$ and the center $\left(\varphi=\Phi_{\mathrm{C}}=0\right)$ of the symmetry section must be identically zero while the value of $J_{z}$ are peaked. This approximation is more accurate the further the flow is axially from the magnetizer source, i.e. the far-field effect. Here, the center, $\Phi_{C}=0$, is defined to be directly under the axial center-line of a pole piece; the values for $\Phi_{\mathrm{E}}$ are $\pi / \mathrm{n}$. Also, note that at the two midpoints between the center and ends $\left(\varphi= \pm \Phi_{\mathrm{M}}= \pm 1 / 2 \Phi_{\mathrm{E}}\right)$, the value of $\mathrm{J}_{\varphi}$ is roughly peaked (and opposite) while the value of $\mathrm{J}_{\mathrm{Z}}$ is minimal (and close to zero).

Based on the symmetry of this system, the circumferential distribution for $\mathrm{J}_{\varphi}$ would need to be an odd function of the coordinate $\varphi$ that satisfies the above boundary conditions. To first order, we can approximate this odd function as

$$
F(\varphi) \approx F_{0} \sin (n \varphi)
$$

The actual function need not be a sine wave, but its function and derivatives should behave similarly. Using separation of variables, the function for $\mathrm{J}_{\varphi}$ can be written as 


$$
J_{\varphi}(\varphi, z)=J_{0} J(z) \sin (n \varphi)
$$

where $\mathrm{J}(\mathrm{z})$ is only a function of the axial coordinate, $z$. Now, the circumferential distribution for $\mathrm{J}_{\mathrm{Z}}$ must be an even function in $\varphi$. This is satisfied by substituting Equation 8 into Equation 2, where upon integrating $\mathrm{J}_{\varphi}$ with respect to $\varphi$ an even function is naturally obtained for $\mathrm{J}_{\mathrm{Z}}$.

Equation 2 can now be solved to determine $J(z)$ and so both $J_{\varphi}(\varphi, z)$ and $J_{z}(\varphi, z)$. Substitution of Equation 8 into Equation 2 yields

$$
\frac{\partial J_{Z}}{\partial z}=-\frac{\partial J_{\phi}}{r \partial \phi}=-\frac{n}{r} J_{0} J(z) \cos (n \varphi)
$$

Integration with respect to $z$ yields determines that

$$
J(z)=e^{-\left(\frac{n}{r}\right) Z}
$$

Therefore,

$$
J_{\varphi}(\varphi, z)=J_{0} \sin (n \varphi) e^{-\left(\frac{n}{r}\right) Z}
$$

and the exponential behavior of the current density as a function of axial distance is revealed. The value of $\mathrm{J}_{\mathrm{o}}$ is given by Equation 3 and is

$$
J_{0}=\sigma \omega r \beta M_{0}
$$

where $\mathrm{M}_{\mathrm{o}}$ is the pole piece's magnetizing strength and $\beta$ is a constant between 0 and 1 that determines how much radial flux is coupled into the pipe wall.

Now, the circumferential component of Equation 1 yields:

$$
\frac{\partial B_{R}}{\partial z}=4 \pi \mu J_{\phi}=-4 \pi \mu J_{0} F(\varphi) e^{-\left(\frac{n}{r}\right) z}
$$

Solving for $\mathrm{B}_{R}(\mathrm{z})$ and substituting Equation 12 into Equation 13 yields the following relationship for the radial field

$$
B_{R}=2 \pi \frac{\beta}{n}\left(\frac{d}{\delta}\right)^{2} M_{0} F(\varphi) e^{-\left(\frac{n}{r}\right) Z}
$$

where $\delta$ is the classical skin depth and $\mathrm{d}$ is the pipe diameter, $d=2 r$. Again, the exponential characteristic of the magnetic field is revealed. Because

$$
\nabla \bullet \vec{B}=0
$$

all three components of the magnetic field will have identical exponential decay along the pipe axis, at least in this approximation.

As a side note, in this first order approximation, the peak amplitudes of the axial and radial current densities are equal. In actuality, the ratio of radial to axial current densities is slightly less than unity because there is also a small amount of radial current flow.

The same will be true for the radial to axial peak magnetic field amplitudes. In fact, the peak amplitude of the magnetic field as a function of axial position is given by 


$$
B_{p k}(z) \propto \frac{\beta}{n}\left(\frac{r}{\delta}\right)^{2} M_{0} e^{-\left(\frac{n}{r}\right) Z}
$$

Notice, the peak amplitude of the magnetic field is proportional to the magnetizing strength of the pole piece (and the coupling factor) and the square of the ratio of the pipe diameter to classical skin depth, and inversely proportional to the number of pole pieces. Also, the exponential decay constant, given by the ratio $\mathrm{n} / \mathrm{r}$, will cause greater decay for smaller pipe diameters and a higher number of pole pieces. This first order approximation suggests that the decay rate is basically geometry dependent.

The modeling results also support these conclusions. Based on the finite element models, the following conclusions were supported: (1) the amplitude of the fields were mostly linear with rotational frequency; (2) the fields decreased exponentially independent of frequency, magnetizing strength, and pipe material property, (3) a larger number of poles (e.g., a 4- vs. 2pole magnetizer) increased the decay rate and decreased magnetic field levels far from the source, and (4) a smaller diameter pipe (e.g., 12-in vs. 24-in diameter pipe) increased the decay rate and decreased the magnetic field levels far from the source.

It should be noted that in the above approximation and in the finite element model, the magnetic permeability was assumed to be linear and isotropic. In actuality it is neither, and so some variations about this behavior can be expected. Also, the approximation is not valid near the magnetizer's pole pieces, i.e., close to the source. Here, the near-field effects are predominating. This fact results in a different near-field behavior as can be seen in Figure 2. In Figure 2, the computed axial decay of the axial component is compared with the closed form solution (equation 16) and experimental results. The computed results were obtained using magnetic finite element analysis (FEA). The solution was obtained using a three-dimensional rotational analysis problem solver that could calculate the current generated by a permanent magnet passing a conductor (Opera-3d $\AA$ from Vector Fields, Ltd., Aurora, Illinois). For the experimental results, the rotating magnet assembly was at one axial location while the sensor was moved along the inside surface of the pipe. At discrete locations along the pipe, the amplitude was measured with a Hall Effect sensor. The density of measurements was greater in the near field than the far field due to the nature of the amplitude changes. As illustrated in Figure 2, calculations and experiments show that the magnetic field decay is exponential. The rate of decay in the 12-inch pipe is nominally an order of magnitude per pipe diameter. Experimental results, superimposed on the calculated results, confirm the analytical equation and the finite element calculations. 


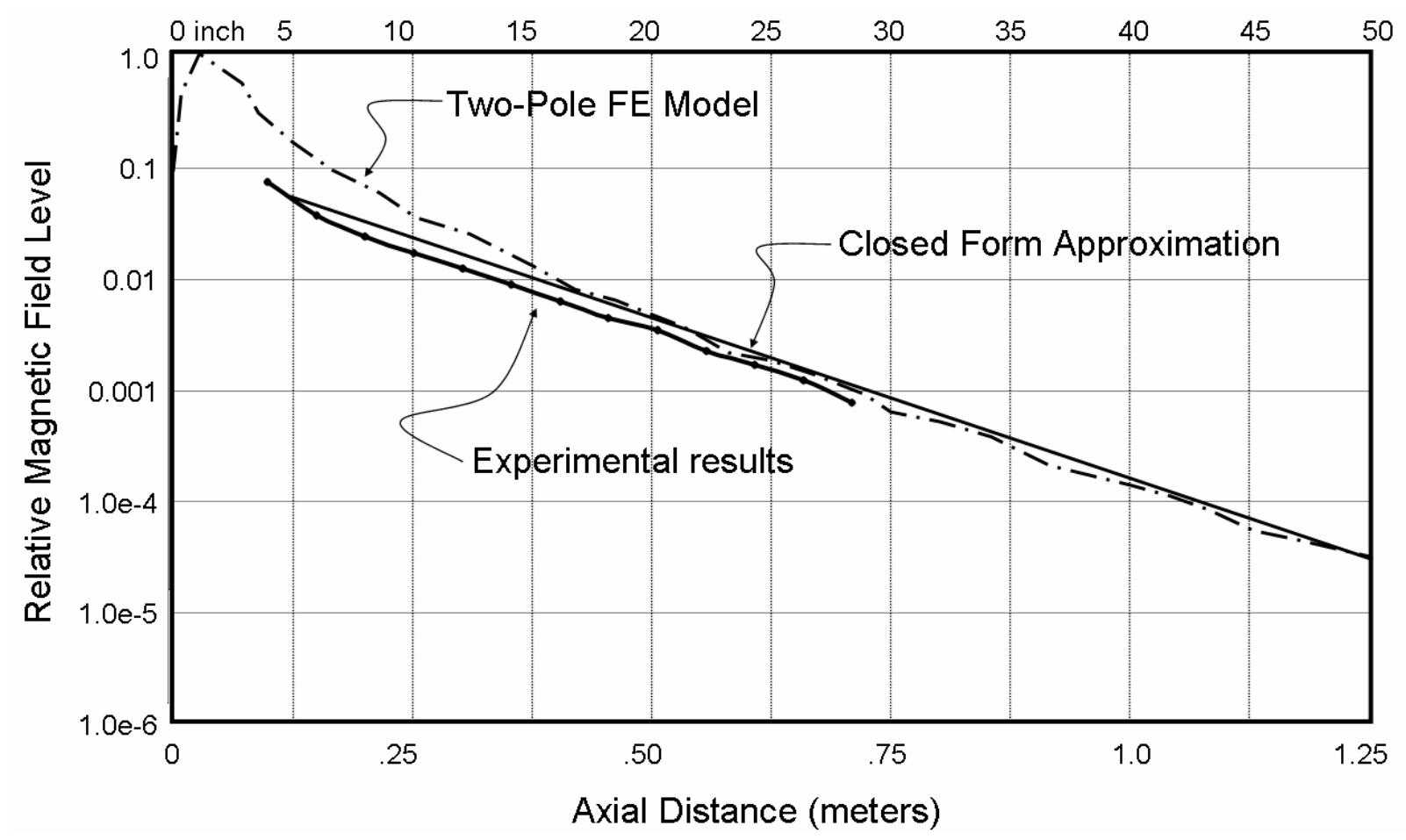

Figure 2. A comparison of the first-order estimate, modeling and experimental results.

\section{Potential Implementations}

Another feature of this implementation is the magnet can be configured to a form factor that enables the tool to pass obstructions within the pipeline. For example, Figure 3 shows a telescopic magnet bar that enable the magnets to retract as they pass over obstructions. Alternatively, Figure 4 shows a hinged structure that enables the bars to bend to pass over obstructions. For the telescoping structure, simple mechanical devices such as worm screws can be used to move the magnets into the proper position for inspection. However, the maximum extent of the telescoping magnetizer in the collapsed position is greater than the hinge configuration. For the hinge configuration, the magnetizer could be designed to fit through obstructions that are less than one-third the pipe diameter. For a two pole magnetizer, the hinged magnetizer could easily pass a plug valve. In the collapsed position, however, the force of attraction of the magnets may be strong and the mechanism to return the magnets to the inspection position might require considerable force. 

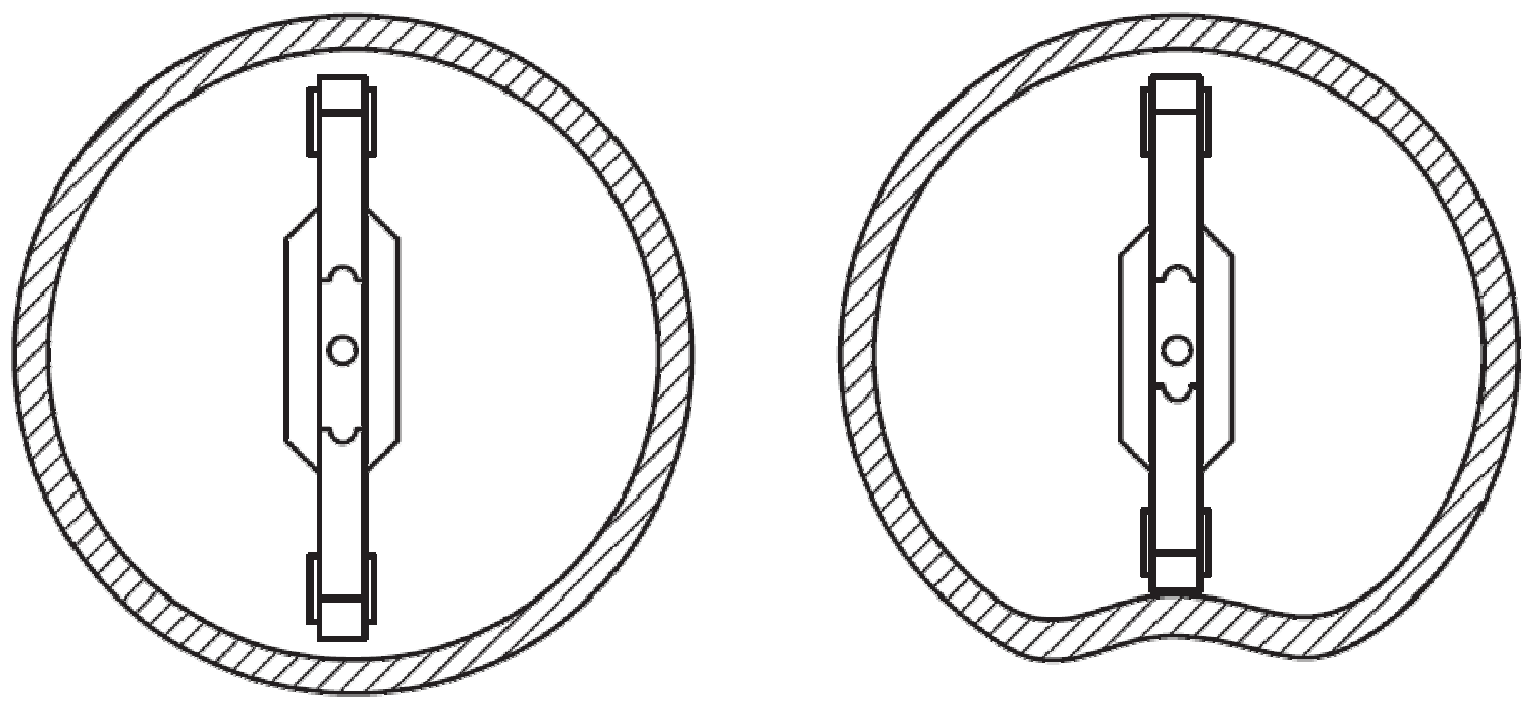

Figure 3. A telescopic magnet bar that enables the magnets to retract as they pass over obstructions
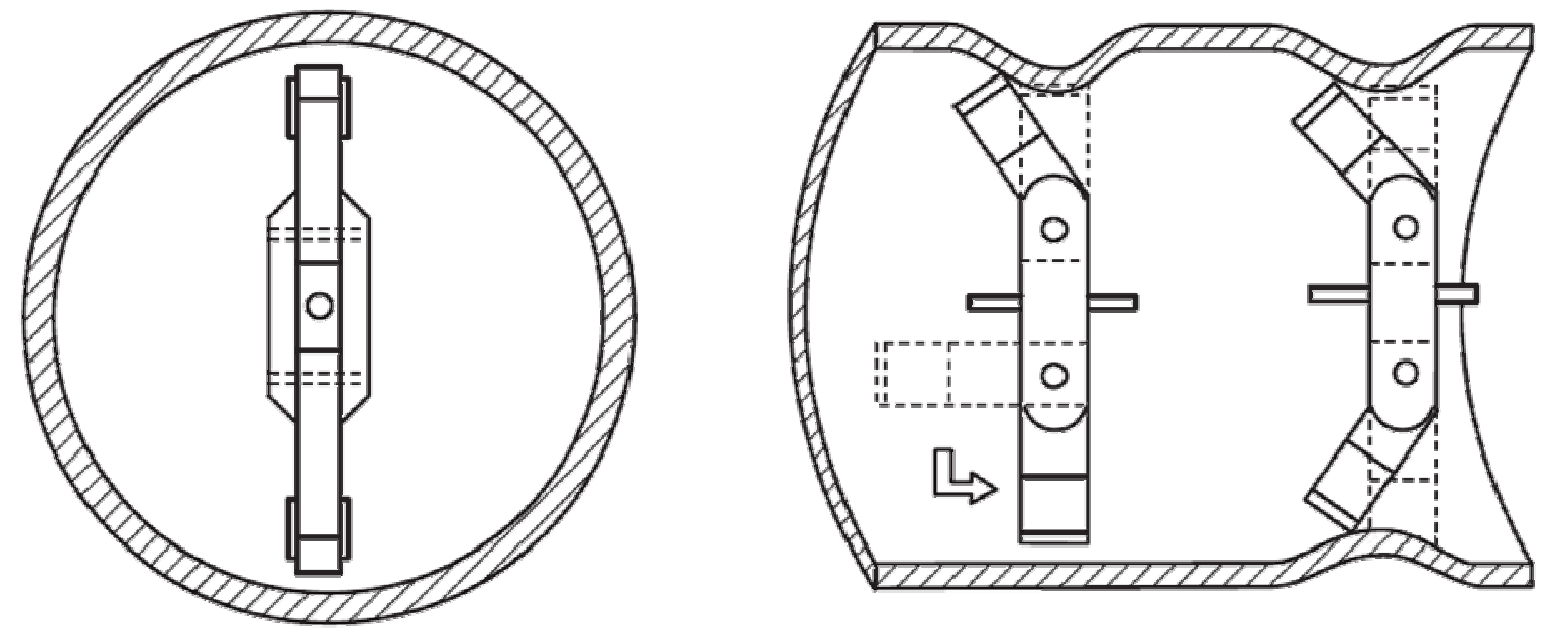

Figure 4. A hinged structure that enables the magnets to retract as they pass over obstructions.

\section{Signal processing}

A rotating permanent magnet is used to induce a magnetic field in the pipe wall under inspection. The resulting eddy currents that flow in the pipe wall produce a secondary magnetic field that can be measured at a sufficient distance from the primary magnetic field. Defects in the pipe wall cause localized changes in flux density in the area of the defect. A Hall sensor located in the area of the secondary field can be used to measure the field disturbance due to the pipe wall defect. Finite-element state modeling of the system has shown that the secondary magnetic field produced is sinusoidal in nature with a fundamental frequency that is given by the rotational speed of the two-pole permanent magnet. Based on an observation of experimental data, the effect of a pipe wall defect on the measured magnetic field is to modulate the amplitude of the sinusoidal signal in a manner that is proportion to the defect size. Calculating the amplitude of 
the measured field strength while the system moves though the pipe can provide information about the location and severity of wall defects.

We propose three potential signal processing approaches for determining the signal amplitude (hence, the pipe wall defect profiles) from measurements of the field strength using a linear output Hall Effect sensor located in the secondary magnetic field. Simulation results are provided that shows the performance of each method with varying simulation parameters (noise, crawler speed, rotor speed, and rotor speed deviation).

The signal processing problem can be stated as follows. We wish to accurately determining the amplitude of the measured magnetic field in the presence of noise. The measured signal can be written as,

$$
x(t)=r(t)+\eta(t)
$$

where, $x(t)$ is the measured signal, $r(t)$ is the sinusoidal signal for which the amplitude is to be estimated, and $\eta(t)$ is the noise component. The sinusoidal signal $r(t)$, can be written as;

$$
r(t)=A(t) \cdot \cos \left(\omega_{c} t+\theta\right)
$$

where, $A(t)$ is the amplitude, which is a function of time (distance along the pipe), $\omega_{c}$ is the angular frequency, and $\theta$ is the phase.

The signal processing problem is to accurately estimate $A(t)$ given measurements of the signal $x(t)$.

\section{Lock-in Amplifier Method (Synchronous AM Demodulation)}

Observing that Equation 18 is in the form of a Double Sideband - Suppressed Carrier, Amplitude Modulated (DSB-SC AM) signal, led to the investigation of AM demodulation techniques to estimate (recover) the signal amplitude $A(t)$. In DSB-SC AM demodulation, a reference sinusoid is used to demodulate a message signal from a carrier. The difficulty with this technique is in generating a reference that is both at the proper frequency and in phase with the input signal. A Phase Locked Loop (PLL) is often used for this purpose. The PLL employs a feedback loop to "lock" onto the input signal and maintain the reference output at the proper frequency and at a constant phase relationship to the input.

A similar device that is employed which is essentially the same method to demodulate the input is known as a lock-in amplifier.

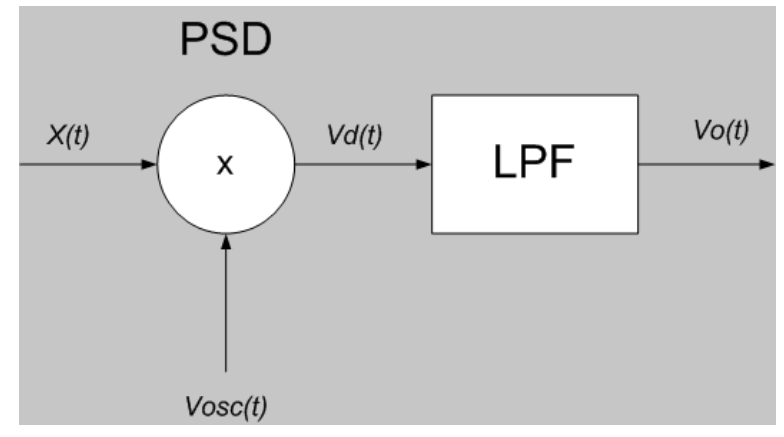

Figure 5. Lock-in Amplifier Block Diagram. 
A block diagram of a lock-in amplifier (LIA) is shown in Figure 5. The LIA consists of a phase sensitive detector (PSD), which is nothing more than a multiplier, and a low-pass filter (LPF). The LIA utilizes a reference oscillator to produce a sinusoidal signal with the same angular frequency as the signal of interest. For synchronous AM demodulation a phase-locked loop (PLL) is employed to generate $\operatorname{Vosc}(t)$ with fundamental frequency $\omega_{c}$ and phase angle $\theta_{o}$, that is related to the phase $\theta$ by a constant. That is, $\left(\theta-\theta_{o}\right)=C$, and ideally $C=0$. The PLL implements closed loop feedback that keeps $\operatorname{Vosc}(t)$ "locked" with the input even for variations in input frequency. For the LIA, the phase of $\operatorname{Vosc}(t)$ is either determined by experiment or $\operatorname{Vosc}(t)$ is derived from the same source as the input. For the experimental method, the oscillator phase is adjusted until maximum output is achieved.

The detected signal can be derived as follows. The oscillator output, with amplitude $A_{\text {osc }}$, is given as

$$
\operatorname{Vosc}(t)=A_{o s c} \cdot \cos \left(\omega_{c} t+\theta_{o}\right) .
$$

Therefore, the output of the PSD is given by

$$
V d(t)=A(t) \cdot A_{o s c} \cdot \cos \left(\omega_{c} t+\theta\right) \cdot \cos \left(\omega_{c} t+\theta_{o}\right)+\eta(t) \cdot A_{o s c} \cdot \cos \left(\omega_{c} t+\theta_{o}\right) .
$$

It is convenient to ignore the term associated with the noise so that,

$$
V d^{\prime}(t)=A(t) \cdot A_{o s c} \cdot \cos \left(\omega_{c} t+\theta\right) \cdot \cos \left(\omega_{c} t+\theta_{o}\right) .
$$

Using a trigonometric identity, Equation 21 can be rewritten as,

$$
V d^{\prime}(t)=.5 \cdot A(t) \cdot A_{o s c} \cdot \cos \left(\theta-\theta_{o}\right)+.5 \cdot A(t) \cdot A_{o s c} \cdot \cos \left(2 \omega_{c} t+\theta+\theta_{o}\right) .
$$

Passing this signal through an ideal low-pass filter (LPF) with a cutoff frequency less than $2 \omega_{c}$ and with bandwidth $W$ that is the bandwidth of $A(t)$ will produce the output

$$
V^{\prime}(t)=.5 \cdot A(t) \cdot A_{o s c} \cdot \cos \left(\theta-\theta_{o}\right) \text {. }
$$

Assuming that $\left(\theta-\theta_{o}\right)=0$, and letting $A_{o s c}=1$, the output is the desired result

$$
V o^{\prime}(t)=\frac{A(t)}{2} .
$$

Taking into account the noise term present in Equation 20, it can be shown that

$$
V o(t)=\frac{A(t)}{2}+\frac{n_{c}(t)}{2},
$$

where, $n_{c}(t)$ is the "in-phase" component of the noise which has the same power content as $\eta(t)$ and for zero mean, white Gaussian noise, the signal to noise ratio is given by

$$
S N R=\frac{P_{A}}{W \cdot N_{o}} .
$$

Here, $P_{A}$ is the power in $A(t), W$ is the bandwidth of the LPF and $N_{o}$ is the variance of $\eta(t)$. 


\section{Vector Subspace Method}

Using a trigonometric identity, Equation 18 can be rewritten as:

$$
\begin{gathered}
r(t)=A(t) \cdot\left[\cos \left(\omega_{c} t\right) \cdot \cos (\theta)-\sin \left(\omega_{c} t\right) \cdot \sin (\theta)\right], \\
r(t)=A(t) \cdot \cos (\theta) \cdot \cos \left(\omega_{c} t\right)-A(t) \cdot \sin (\theta) \cdot \sin \left(\omega_{c} t\right), \\
r(t)=a \cdot \cos \left(\omega_{c} t\right)-b \cdot \sin \left(\omega_{c} t\right) .
\end{gathered}
$$

It follows that,

$$
\begin{gathered}
a=A(t) \cdot \cos (\theta), \\
b=A(t) \cdot \sin (\theta), \\
A(t)=\operatorname{sqrt}\left(a^{2}+b^{2}\right),
\end{gathered}
$$

and

$$
\theta=\arctan (b / a)
$$

After analog-to-digital conversion the measure signal is given as:

$$
x(n)=r(n)+\eta(n)
$$

and

$$
r(n)=a \cdot \sin \left(\omega_{c} n T\right)-b \cdot \cos \left(\omega_{c} n T\right) .
$$

Given the sample time $T$ and $N$ samples, $r(n)$ can be written in matrix-vector form as follows,

$$
\begin{gathered}
{\left[\begin{array}{c}
r(0) \\
r(1) \\
\vdots \\
r(N-1)
\end{array}\right]=\left[\begin{array}{cc}
\sin (0) & -\cos (0) \\
\sin \left(\omega_{c} T\right) & -\cos \left(\omega_{c} T\right) \\
\vdots & \vdots \\
\sin \left(\omega_{c}[N-1] T\right) & -\cos \left(\omega_{c}[N-1] T\right)
\end{array}\right] \cdot\left[\begin{array}{l}
a \\
b
\end{array}\right]} \\
\vec{r}=A \cdot \vec{v} .
\end{gathered}
$$

If we had samples of $r(n)$ we could solve for $\vec{r}$; however, since we only have samples of $x(n)$ we must formulate a different problem. Due to the additive noise, it is unlikely that $\vec{x}$ will lie in the column space of $A$ and, therefore, there will not be a solution $\vec{v}$ such that:

$$
\vec{x}=A \cdot \vec{v}
$$

Instead, we pose the problem as follows: we wish to find $\hat{v}$, such that, $\|A \hat{v}-\vec{x}\|$ is minimized. 
From linear algebra, the solution is given by the least squares projection of $\vec{x}$ onto the subspace spanned by the columns of $A$. That is, the projection of $\vec{x}$ onto the subspace of sinusoidal signals with angular frequency $\omega_{\mathrm{c}}$.

The solution can be found using a pseudo inverse as follows:

$$
\hat{v}=\left(A^{T} A\right)^{-1} A^{T} \cdot \vec{x}
$$

\section{Processing Complications}

Signal Bandwidth. The signal represented by Equation 18 is not a typical AM signal due to the fact that the bandwidth, $W$ of $A(t)$ may be greater than the fundamental frequency $\omega_{c}$ of the carrier signal. The nature of the PM crawler system is such that, the rotational speed of the permanent magnet assembly is slow, on the order of $5 \mathrm{~Hz}$, and for certain (sharp) defects the modulating signal could contain much higher frequency content. This poses a problem for the LIA detection approach. Adjusting the ideal LPF cutoff to be equal $\omega_{c}$ would reject frequencies in the modulating signal that are greater than $\omega_{c}$. The resultant output of the LIA would be a low pass version of $A(t)$ and depending on the amount of signal filtered out could result in a poor estimate. This problem could be eliminated by processing $N$ samples of the input at a time and then computing an $N$-point FFT of this result. The zero frequency component of the FFT would give an estimate of $A(t)$.

Variable Rotor Speed. It is likely that the speed of rotation of the magnet assembly will drift as the crawler moves down the pipe due to changes in drive motor applied voltage as well as other factors. To preclude the need to incorporate tight control on motor speed it is desirable that an updated method be incorporated to accommodate any changes in rotor speed and hence fluctuations in $\omega_{c}$. A sensor could be attached to the rotor to provide an input of rotor speed that could be used to update $\omega_{c}$ periodically. Both of the processing methods discussed above would require this input to account for changes in $\omega_{\mathrm{c}}$ unless a PLL was designed that could be used with the LIA method. It is believed that it would be difficult to design a PLL that would work at such low operating frequencies.

\section{Other Methods}

Another AM demodulation technique that could be employed to recover $A(t)$ is an envelope detector. For the case were $A(t)>0$, for all $t$, the envelope detector would be a valid approach. An envelope detector is nothing more than a rectifier followed by a low pass filter. However, for reasons previously discussed the low pass filter operation would degrade the estimate of $A(t)$. As with the previous methods, the envelope detector method could be employed on $N$ points at a time and an the zero frequency term of an FFT would produce an estimate of $A(t)$. 


\section{Results and Discussion}

\section{Comparison on Theory and Experiment}

Data was acquired to assess the accuracy of the closed form equation for designing rotating exciters and positioning sensors, [Equation 16]. Figure 6 shows how the decay rate is related to both pipe diameter and number of poles. Three configurations were tested:

1. a six-inch $(155 \mathrm{~mm})$ diameter pipe and two-pole magnetizer;

2. a 12-inch $(310 \mathrm{~mm})$ diameter pipe and two-pole magnetizer; and

3. a 12-inch $(310 \mathrm{~mm})$ diameter pipe and four-pole magnetizer.

The wall thickness of both pipe samples was nominally $3 / 8$ inches $(9 \mathrm{~mm})$ and it was assumed that the magnetic permeability and electrical conductivity of both samples were equal. The rotational frequency was five hertz. The rotation speed for the four-pole unit was cut in half to keep the frequency of the inspection current equal to the other two configurations. The plots in Figure 6 show that the decay rate is similar for the six-inch magnetizer with two poles and the 12-inch magnetizer with four poles; only the initial amplitude of the smaller diameter magnetizer is lower. The decay rate of the 12-inch magnetizer with two poles is nominally half of the other two. For the four-pole configuration in the 12-inch pipe, two bars were added to the two-pole magnet assembly and the polarity of the magnets assigned appropriately.

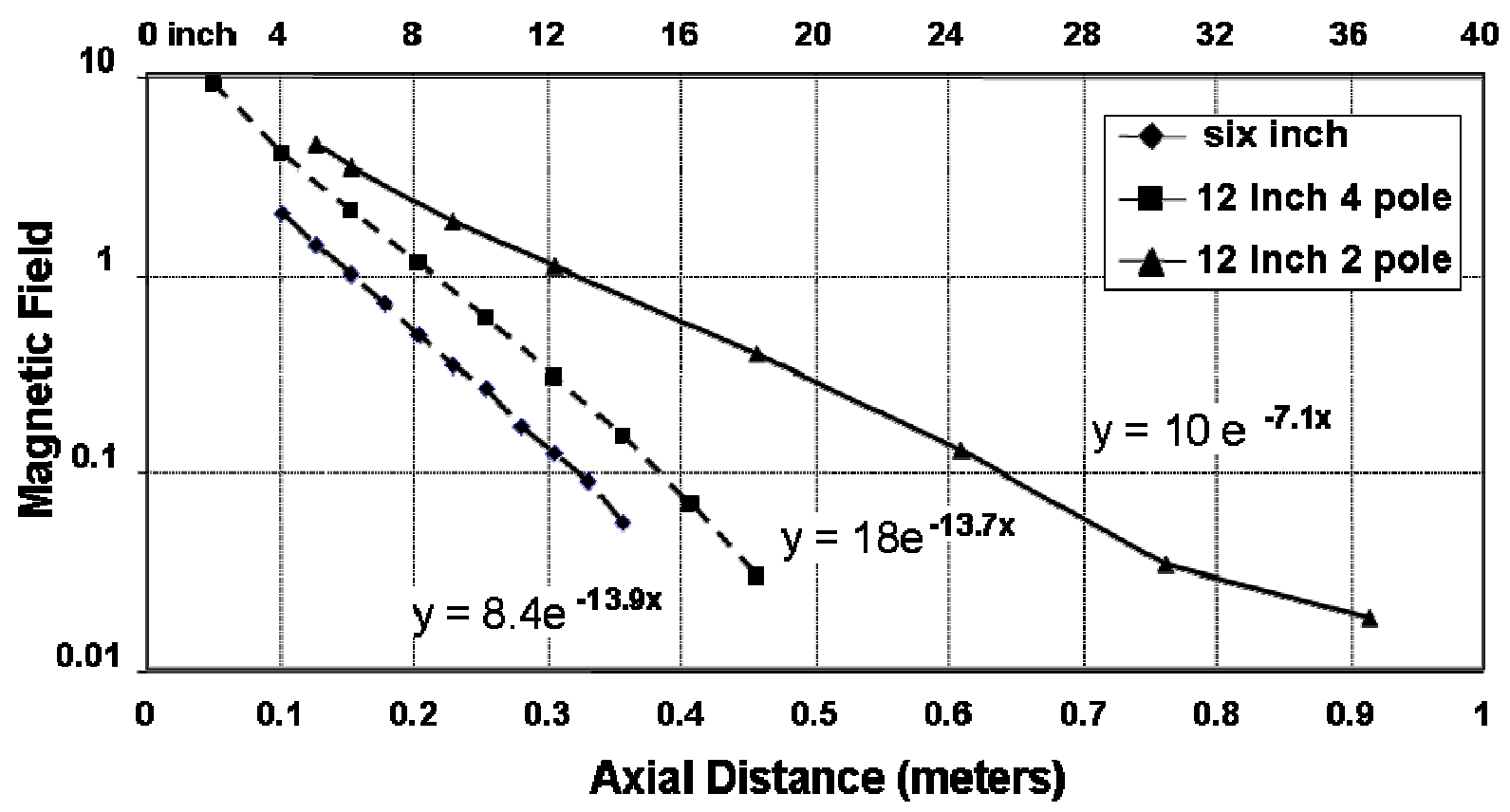

Figure 6. Experimental results showing the decay rate is related to both pipe diameter and number of poles 
Table 1. A comparison between calculated and experimental measured decays.

\begin{tabular}{|c|c|c|c|c|c|}
\hline \multirow{2}{*}{ Description } & \multicolumn{2}{|c|}{ Diameter } & \multirow{2}{*}{$\begin{array}{c}\text { Number of } \\
\text { poles }\end{array}$} & \multicolumn{2}{c|}{ Pole Pairs / Radius } \\
\cline { 2 - 3 } \cline { 5 - 6 } & Inches & Meters & Calculated & Experiment \\
\hline 12 inch 2 pole & 12 & 0.305 & 2 & 6.6 & 7.1 \\
\hline 12 inch 4 pole & 12 & 0.305 & 4 & 13.1 & 13.7 \\
\hline 6 inch 2 pole & 6 & 0.152 & 2 & 13.1 & 13.9 \\
\hline
\end{tabular}

\section{Signal Processing}

We performed several simulations to evaluate the performance of the methods discussed above. The parameters that were investigated for the purpose of simulation were; the rotor speed [Hz], the crawler speed [in/sec], the Signal-to-Noise Ratio (SNR) [dB], the pipe defect size [in], the rotor frequency deviation [Hz/in] and the number of cycles processed [cycles]. The number of cycles determines the number $N$ of points processed at a time to compute the result. The specific parameters used for each simulation are summarized in Table 2. Simulation results are presented for each case along with a brief discussion of the significance of the results.

Table 2. Simulation parameters.

\begin{tabular}{|l|c|c|c|c|c|c|}
\hline Parameter & SIM 1 & SIM 2 & SIM 3 & SIM 4 & SIM5 & SIM6 \\
\hline Rotor Speed [Hz] & 5 & 5 & 5 & 5 & 5 & 5 \\
\hline Crawler Speed [in/sec] & 3 & 3 & 1 & 3 & 1 & 1 \\
\hline SNR [dB] & inf & 20 & 20 & Inf & 20 & 20 \\
\hline Defect Size [in] & 2 & 2 & 2 & 2 & 2 & 2 \\
\hline Max Frequency Deviation[Hz/in] & 0 & 0 & 0 & .05 & .05 & .05 \\
\hline Num Cycles & 1 & 1 & 2 & 1 & 2 & 1 \\
\hline
\end{tabular}

The pipe profile $A(t)$ was modeled with a square wave to simulate defects with sharp edge transitions and with a specific depth. This allowed us to analyze the detection performances based on defect size and severity. Each simulation result shows the pipe wall profile, the modulated sinusoid $r(t)$ and the results for the three detection methods.

SIM 1 - Ideal Estimation. This case is the ideal case where there is no additive noise (infinite SNR) present in the received signal $x(t)$ and no frequency deviation (constant rotor speed) as the 
crawler moves along the pipe. The number of cycles to process for this case was set to 1.0. The results are shown in Figure 7. All three detectors (LIA, Subspace and Envelope) achieve perfect results. The filter effect of processing $N$ samples at a time is also evident by the transients observed as the pipe wall transitions sharply from normal to defect and then back. The filter effect can be reduced by reducing the number of points $N$, however; a minimum number of points (corresponding to cycles of $r(t)$ ) is required to produce an accurate result.

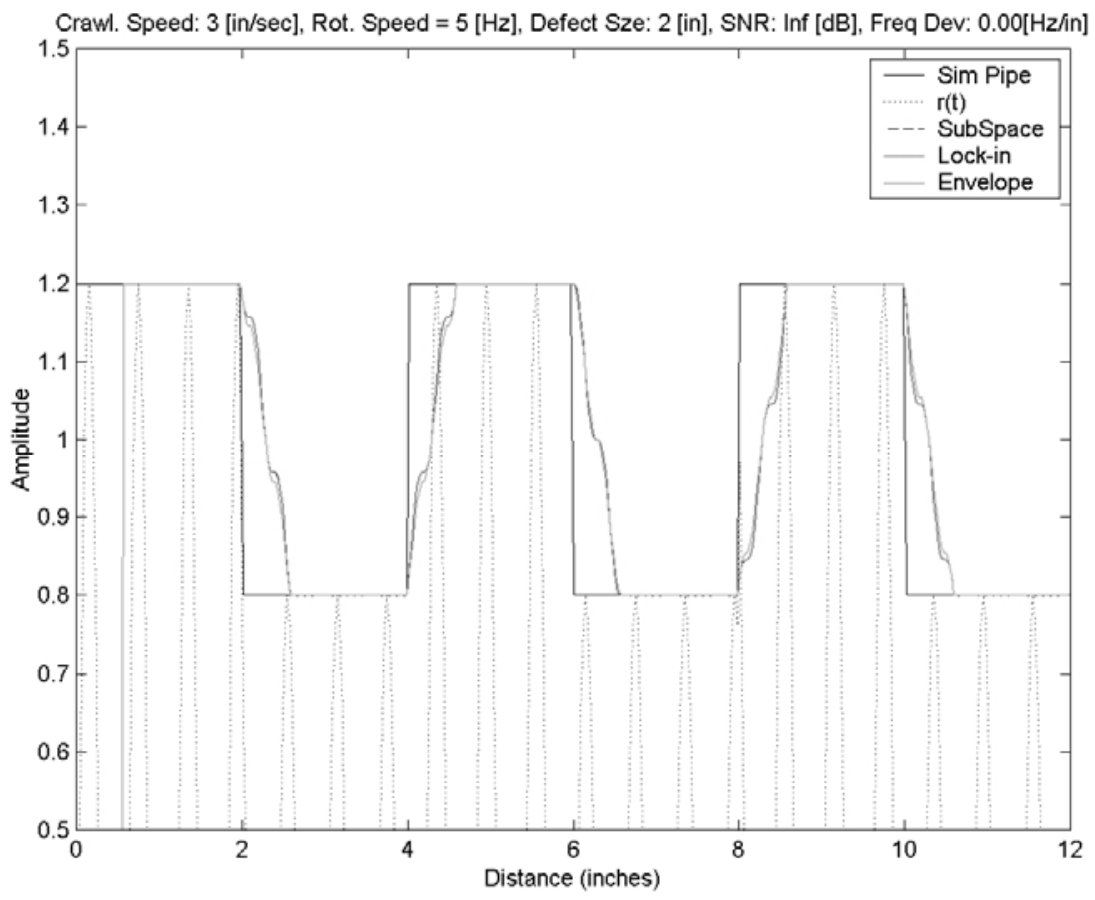

Figure 7. Simulation 1 Results

SIM 2 - Additive Noise Simulation. In this case Gaussian noise was added to $r(t)$ such that the SNR was 20 [dB]. This SNR was determined to be comparable to the SNR of measured data taken with a prototype PM crawler system. The SNR achieved in a real system could be worse and is largely dependent upon the rotating magnet configuration. Higher SNR is a function of how close the permanent magnets are to the pipe wall. However, the closer the magnets are the more torque (and ultimately the more power) required to turn the assembly. Since the PM Crawler will operate from a battery source, it is expected that the magnets may need to be backed away from the pipe wall to guarantee enough battery life for an entire inspection run. The results shown in Figure 8 indicate that additive noise at a level of $20[\mathrm{~dB}]$ does not significantly reduce the detector performance.

For SNR below $20[\mathrm{~dB}]$ the detectors performance can be improved by increasing the number of samples $N$ processed. However, the cost of increasing $N$ is to reduce the resolution (due to the filter effect) of the detectors, that is, the smallest defect that can be resolved. This effect could be countered by decreasing the crawler speed such that there are more rotor cycles per distance. 


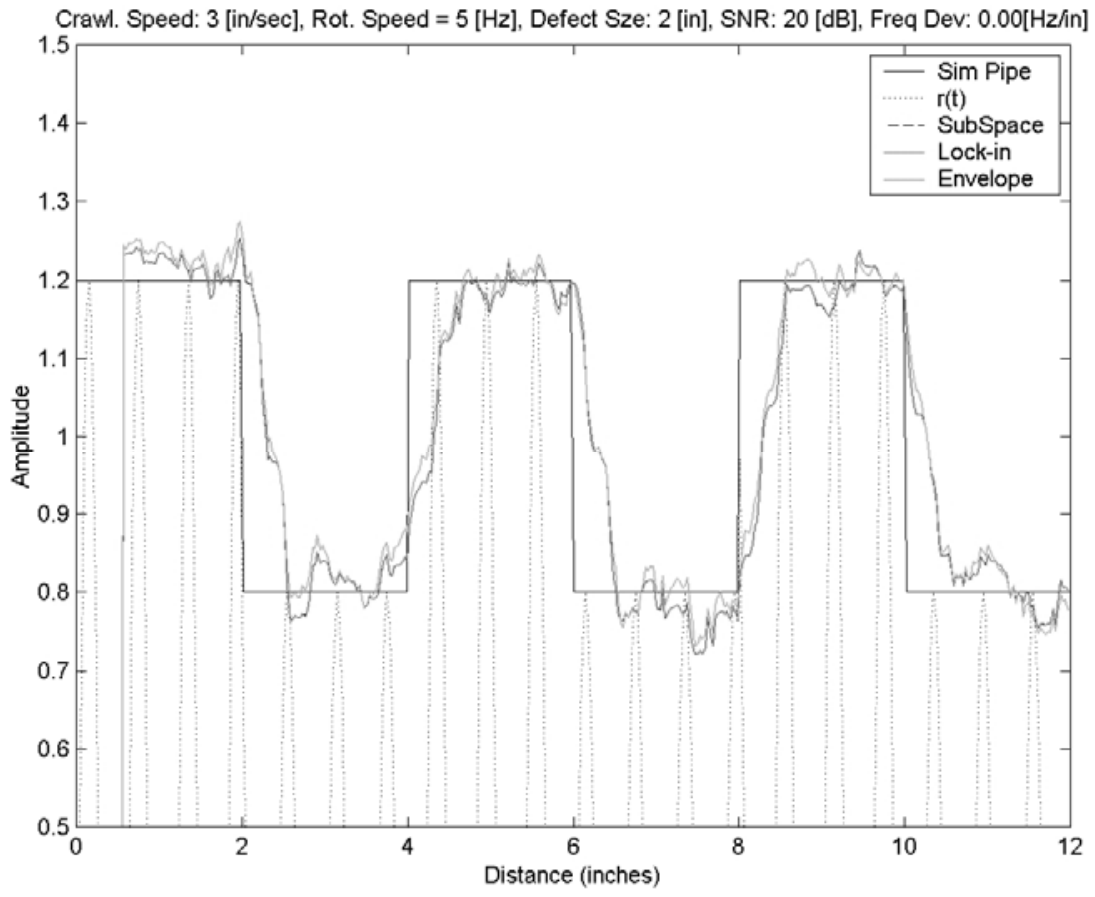

Figure 8. Simulation 2 Results

SIM 3 - Additive Noise - Reduced Crawler Speed. Figure 9 shows the third simulation exploring the affect of additive noise and reduced crawler speed. In this case the crawler speed has been reduced and $N$ has been increased to improve the detection performance while maintaining nearly the same detection resolution. 


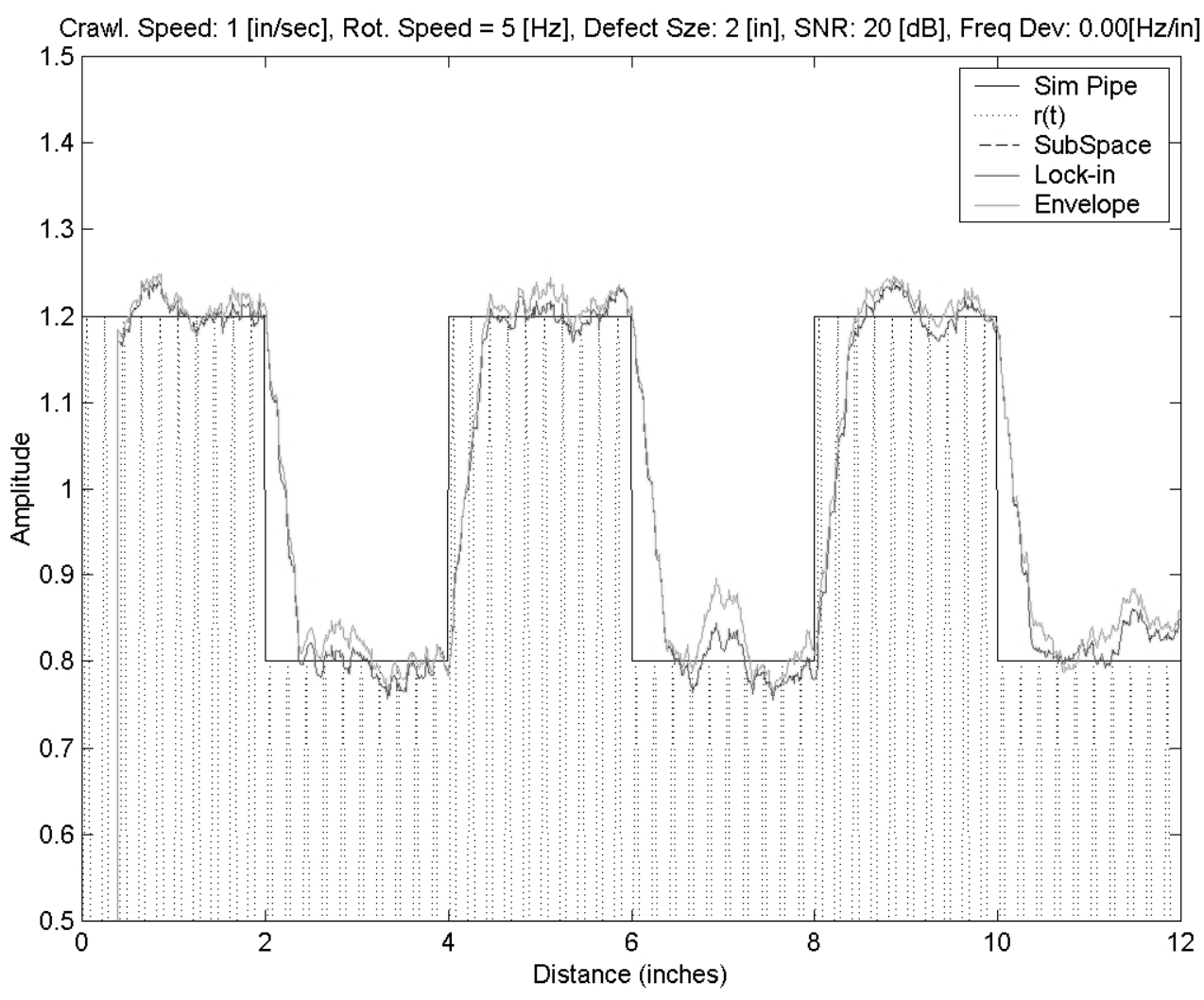

Figure 9. Simulation 3 Results

SIM 4 - Variable Rotor Speed. Figure 10 shows the of the fourth simulation, exploring the affect of variable rotor speed. For this case there is no additive noise present in $x(t)$, instead we have allowed the rotor speed to deviate linearly as a function of distance along the pipe wall. For this case the deviation is $.05[\mathrm{~Hz} / \mathrm{in}]$ which results in a $0.6[\mathrm{~Hz}]$ frequency deviation from left to right in Figure 10. In this case we are applying an update in the signal processing to accommodate the changing rotor speed. As can be seen, the frequency deviation does affect detector performance. The estimates of $A(t)$ oscillate around the true value and the amplitude of oscillation is a function of how fast the rotor speed is changing. For the LIA and Subspace methods this is due to the use of the mean measured frequency for all $N$ points to process the signals. 


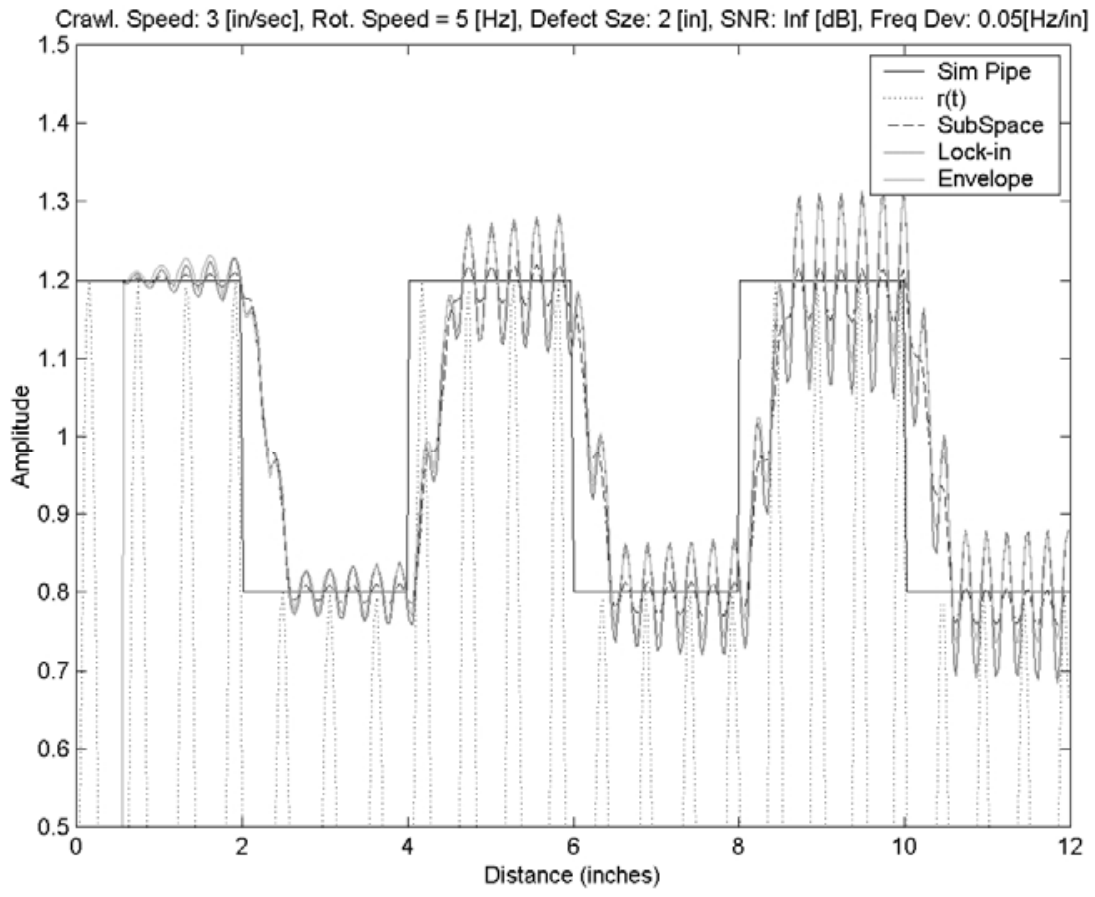

Figure 10. Simulation 4 Results

SIM 5 - Variable Rotor Speed and Reduced Crawler Speed. Figure 11 shows the fourth simulation, exploring the affect of variable rotor speed and reduced crawler speed. In this case we have reduced the crawler speed and are processing over two full cycles. As was expected the LIA and Subspace methods have degraded. Processing over more points means that there will be greater error between the mean measured frequency (which is used to process all $\mathrm{N}$ points) and the actually frequency of $r(t)$ which is changing over all $N$ points. An interesting observation is that the Envelope detector performance does improve. This is because the Envelope detector does not require the frequency measurement input. The envelope method requires no reference signal at all. 


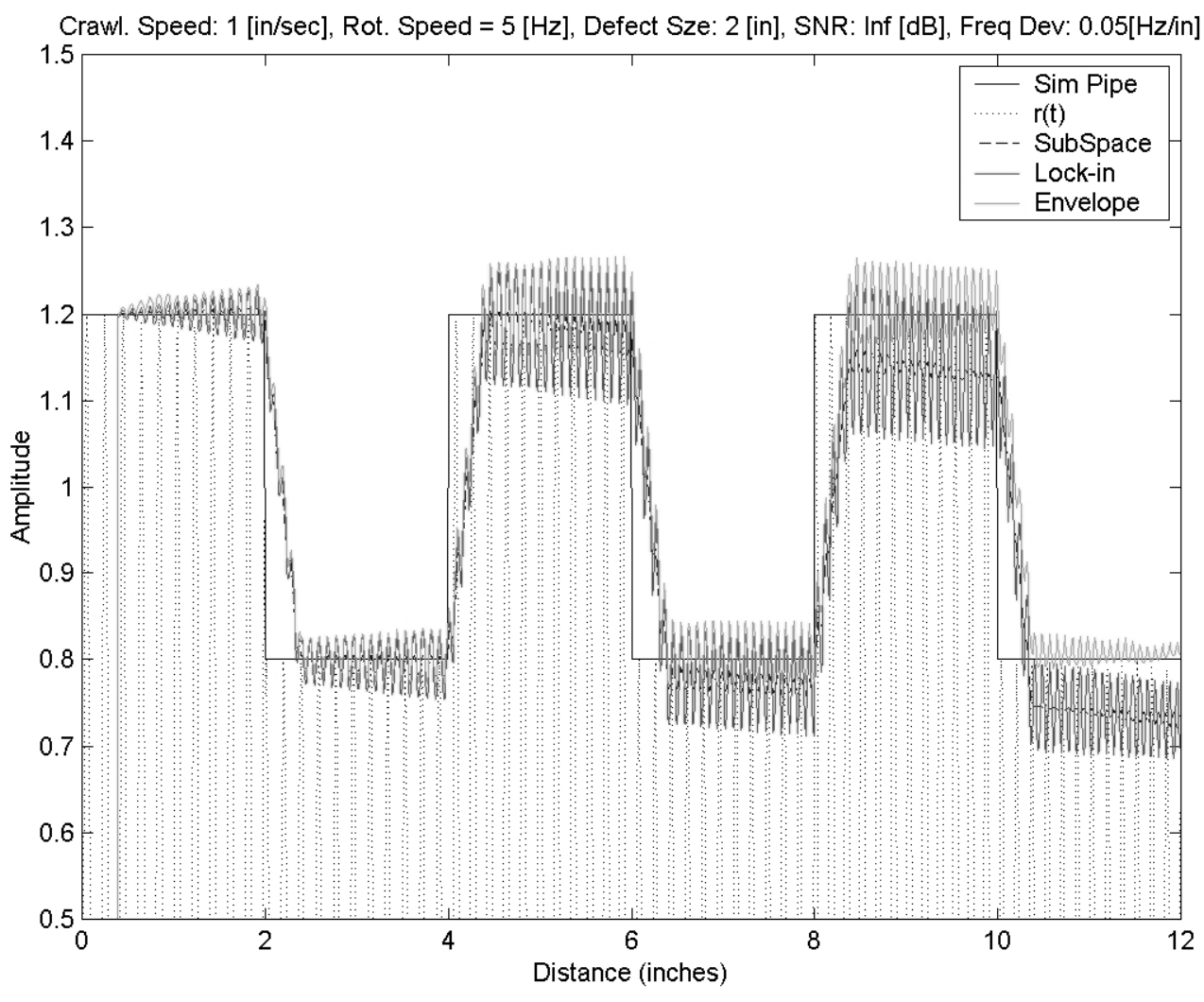

Figure 11. Simulation 5 Results

SIM 6 - Variable Rotor Speed and Additive Noise. Figure 12 shows the results for both varying rotor speed and additive noise. All three methods achieve similar results. In fact for all of the cases presented here the results for each method are comparable. More analysis should be performed to investigate the limitations of each method. Based on the observations of this analysis the Envelope detection method would be preferred due to the simplicity of implementation and the lack of need for a reference input. However, we have made assumptions about $A(t)$ that may not hold for real data. 


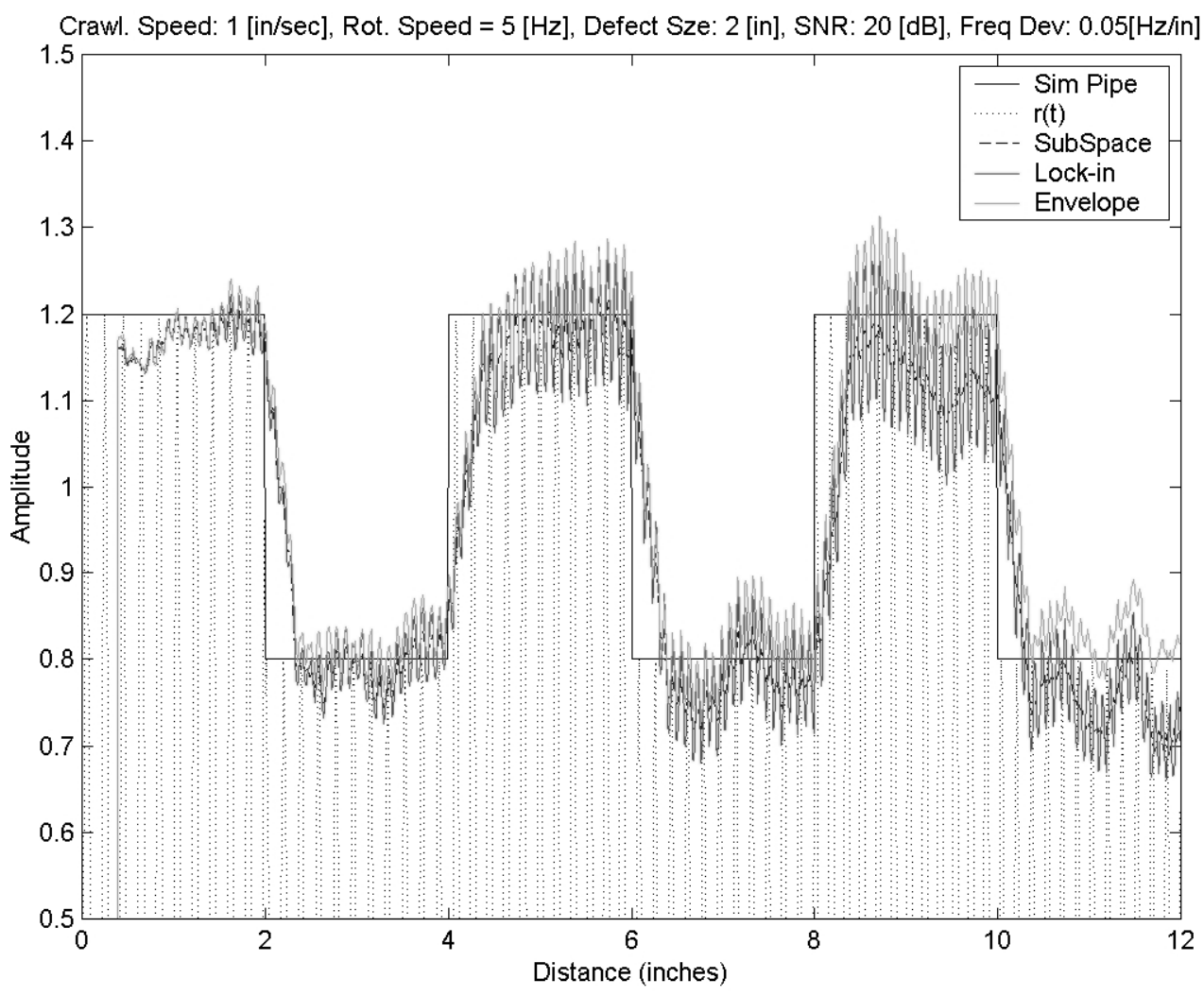

Figure 12. Simulation 6 Results 


\section{Discussion}

The approach being developed has many advantages for inspection of nonpiggable pipelines. First, the two-pole permanent magnet can be closer to the pipe, producing stronger currents in the pipe wall than the commonly used concentric exciter coil. This arrangement will lead to stronger signals at the receiver. The two-pole magnetizer has the form factor to pass rectangular obstructions such as plug valves. Each permanent magnet pole can be hinged to allow it to pass pipeline obstructions. For traditional concentric coil systems, the diameter of the exciter coil must be small enough to pass the largest obstruction. Although the RFEC technique works for exciter coils that have significant separation from the pipe, the induced currents in the pipe are locally weaker and more dispersed, causing a weaker signal at the receiver. Another way to boost signals at the receiver is to apply more current to the exciter coil, but this is not practical on autonomous vehicles. For the rotating permanent magnet exciter, the electrical power consumption should be dramatically less. Instead of continuously providing amps of power to energize the exciter coil, an efficient motor can be used to rotate the assembly. The motor will need a strong starting torque to overcome the static attraction force of the permanent magnets, but once the assembly is rotating, the power consumption is expected to reach a reasonable level.

\section{Conclusions}

A new inspection method is being developed that has excellent potential for unpigable pipelines. A rotating permanent eddy current exciter produces strong magnetic fields at the receiver. The theoretical equations derived from first principles matches the experiments extremely well. This technique has the potential to use less electrical power than coil-based eddy current systems such as remote field eddy currents (RFEC). The prototype unit produces strong eddy currents in the pipe wall. At distances of a pipe diameter or more, the currents flow circumferentially as confirmed by experiments and finite element modeling. These circumferential currents are deflected by pipeline anomalies such as corrosion and axially aligned cracks. Currents are detectable with a simple Hall Effect sensor at distances up to three pipe diameters away, though sensor placement at a pipe diameter away appears to more practical.

This approach has many advantages for inspection of nonpiggable pipelines. The two-pole permanent magnet configuration has a form factor capable of passing rectangular obstructions such as plug valves. The rotating magnet eddy current exciter, although in the early stages of development, has the potential to perform as well as magnetic flux leakage and remote field eddy current inspection technology.

The theoretical work and signal processing investigations performed in this quarter will serve as the foundation for further development of this new inspection methodology. 